TENDENCIAS

Revista de la Facultad de Ciencias

Económicas y Administrativas.

Universidad de Nariño

ISSN-E 2539-0554

Vol. XXIII No. 1 - 1er Semestre 2022

Enero - Junio - Páginas 200-224

\title{
DIVULGACIÓN DE LA INFORMACIÓN AMBIENTAL EN PÁGINAS WEB EN EMPRESAS COTIZADAS CHILENAS
}

\section{DISCLOSURE OF ENVIRONMENTAL INFORMATION ON WEBSITES IN CHILEAN LISTED COMPANIES}

\section{DIVULGAÇÃO DE INFORMAÇÕES AMBIENTAIS EM WEBSITES DE EMPRESAS LISTADAS NO CHILENO}

Fernando Morales Parada; Doris Vergara Puentes; Vanessa Jara Rodríguez

\footnotetext{
Doctor en Contabilidad y Finanzas, Universidad de Zaragoza. Profesor Asociado, Departamento de Administración y Auditoría, Universidad del Bío-Bío. ORCiD: 0000-0001-9046-5407.

E-mail: fmorales@ubiobio.cl, Chile.
}

Contadora Auditora, Universidad del Bío-Bío. Auditor, Universidad del Bío-Bío. ORCiD: 00000002-8926-7216. E-mail: doris.vergara1601@alumnos.ubiobio.cl, Chile.

Contadora Auditora, Universidad del Bío-Bío. Auditor, Universidad del Bío-Bío. ORCiD: 00000002-1211-4385. E-mail: vanessa.jara1601@alumnos.ubiobio.cl, Chile.

Recibido: 21 de abril de 2021

Aprobado: 30 de noviembre de 2021

DOI: https://doi.org/10.22267/rtend.222301.188 


\title{
Resumen
}

No cabe duda de que la divulgación en temas de sostenibilidad es aporte a la problemática mundial medioambiental. En este sentido, este trabajo presenta un análisis de la divulgación de información relacionadas con los Objetivos para el Desarrollo Sostenible (ODS) en las páginas web de 107 compañías chilenas utilizando diferentes variables recogidas de forma dicotómica desde los sitios web corporativos a partir de una lista de chequeo para determinar un índice que permita medir la revelación de información relativa al medioambiente bajo un contexto de sostenibilidad, a partir de cinco índices propuestos. Entre los resultados observamos que las empresas divulgan más respecto a su gobierno corporativo y en menor medida en condiciones laborales. Asimismo, se observa que el sector minero presenta una mejor divulgación promedio, sin embargo, la industria agro-silvícola aún tiene mucho por mejorar en esta materia y las empresas de mayor tamaño y mejor EBIT igualmente se relacionan directamente con la mayor divulgación en internet de informaciones evaluadas.

Palabras clave: Chile; divulgación; internet; medioambiente, sostenibilidad.

JEL: M20; M14; M49; Q56; Q59

\begin{abstract}
There is no doubt that the disclosure on sustainability issues is a contribution to the global environmental problem. In this sense, this work presents an analysis of the disclosure of information related to the SDGs on the websites of 107 Chilean companies using different variables using different variables dichotomously from corporate websites from a checklist to determine an index to determine the disclosure of information related to the environment under a framework of sustainability, based on five indices proposed. In some results, we observe that companies disclose more about corporate governance and less about workers. Likewise, we observe that the mining sector presents a better average disclosure, however, the agricultural and forestry industry still has much to improve in this matter and the larger companies and better EBIT are directly related to the greater disclosure of information on the internet evaluated.
\end{abstract}

Keywords: Chile; disclosure; environment; internet; sustainability.

JEL: M20; M14; M49; Q56; Q59 


\section{Resumo}

Não há dúvida de que a divulgação de questões de sustentabilidade contribui para os problemas ambientais globais. Nesse sentido, este trabalho apresenta uma análise da divulgação de informações relacionadas ODS nas páginas da web de 107 empresas chilenas a partir de diferentes variáveis usando diferentes variáveis coletadas dicotomicamente de sites corporativos a partir de uma lista de verificação para determinar um índice para determinar a divulgação de informações relacionadas ao meio ambiente em um marco de sustentabilidade, com base em cinco índices propostos. Os resultados, observamos que as empresas divulgam mais sobre sua governança corporativa e, em menor medida, sobre as condições de trabalho. Da mesma forma, observamos que o setor de mineração apresenta melhor evidenciação média, porém, a indústria agro-florestal ainda tem muito a melhorar nesse quesito e empresas maiores e melhor EBIT também estão diretamente relacionados à maior divulgação na internet.

Palavras-chave: Chile; divulgação; internet; meio ambiente; sustentabilidade.

\section{JEL: M20; M14; M49; Q56; Q59}

\section{Introducción}

La temática ambiental está cada vez más presente en distintos ámbitos de la sociedad debido a que cada vez se está generando una mayor conciencia por parte de las personas, medios y empresas de lo que se denomina "Cambio Climático" con múltiples campañas que de distintas formas buscan generar una disminución de las externalidades negativas que producen las actividades empresariales para el planeta. Por lo mismo el auge de los reportes de sostenibilidad ha sido creciente, lo que queda demostrado en el informe de KPMG (2020), advirtiendo que esta desalineación con la práctica global aceptada en sí misma plantea riesgos para las empresas que no informan respecto de que hay empresas que no entran en esta práctica de la divulgación, pero los líderes de esas empresas también deben ser conscientes de que los informes de sostenibilidad no se pueden resolver fácilmente de la noche a la mañana con una solución rápida.

Al tener la parte social como un aspecto importante que se encuentra constantemente interrelacionada con la firma, es fundamental saber que las entidades estarán siempre a los ojos de una constante evaluación por parte de ella, lo que hace que la empresa pase a depender del 
juicio social a la hora de actuar, lo que va acorde con la Teoría de la Legitimidad siguiendo las ideas de Vargas (2008), que en pocas palabras se refiere a que las razones de la divulgación medioambiental por medio de las empresas es por el afán de legitimar su existencia en el seno de la sociedad, lo que muchas veces supera el ámbito legal, aunque incluso en ocasiones, puede encontrarse reporte de información no fidedigna por el afán de mantener un estatus ante el entorno social (Carqués, 2007).

Este trabajo se enfoca en la divulgación de la información medioambiental por medio de las páginas web de las empresas de distintos sectores empresariales, debido al aumento de la conciencia de esta temática y en el futuro se planean realizar muchas acciones para mejorar el comportamiento con respecto al cambio climático, lo que se puede traducir en mayor grado de regulación, por lo tanto es importante la aparición de una preocupación actual no sólo por las emisiones directas que genera la empresa sino que en un contexto amplio sobre la responsabilidad social que deben tener al desempeñar sus actividades. Internet en este sentido, cumple las condiciones y características ideales para una mejor comunicación, más aun sabiendo que el proceso de incrementar el reporte vía informes de sostenibilidad no es tan rápido. En este sentido, organismos como el International Accounting Standard Committe (IASC, 1999: actualmente IASB) y la Asociación Española de Contabilidad y Administración de Empresas (AECA, 2002) entre los seminales, hasta el Institute of Chartered Accountants of Scotland (ICAS, 2018) y la firma consultora EY (2017) entre los más recientes.

Sin embargo, Hernández y Yagui (2021) advierten que en Latinoamérica las indagaciones evidencian que las empresas con mayor impacto ambiental y social en sectores como el minero, energía, industrial y agroindustria con una importante influencia institucional de organizaciones locales y transnacionales, han desarrollado la información de sostenibilidad, pero que no llega a los niveles internacionales apoyados en otros autores.

El propósito de este trabajo es evaluar la información que proporcionan una muestra de empresas cotizadas chilenas en sus respectivas páginas web, calificando su contenido medioambiental, social y de gobernanza con relación a los Objetivos para el Desarrollo Sostenible, entre otros contenidos que sean relativos a los antes mencionados La evaluación inicial se medirá con el 
método dicotómico para obtener índices de medición de la revelación para posteriormente obtener los análisis estadísticos y sus conclusiones.

\section{Marco teórico}

La transparencia corporativa según Cabrera (2008) es un intento deliberado por parte de la organización de crear un entorno de confianza en el que promocionar el acceso libre a la información, la comunicación abierta y la participación de todos los profesionales en la toma de decisiones, siendo su objetivo comunicar información a los distintos grupos de interés.

Como se nombró anteriormente, este principio de transparencia ha sido reconocido por distintas organizaciones que han emitido múltiples recomendaciones respecto a este concepto, entre ellas la Organización para la Cooperación y Desarrollo Económico (OCDE, 2015) y la federación Internacional de contadores (IFAC, 2018) destacando la importancia sobre todo después de numerosos escándalos relacionados con corrupción en el manejo de información en distintos países, incitando que cada vez más grupos de interés estén atraídos en el acceso de información, para poder hacer su propia determinación a partir de lo que las entidades se encuentran divulgando. (Briano y Rodríguez, 2013).

Al respecto Sandoval (2015) afirma que la Transparencia es la acción que genera el efecto de "apertura"; es decir referirse a una transparencia en los procesos, datos, archivos, la información gubernamental que ha estado guardada, y es importante distinguir este concepto de lo que se refiere una "apertura de gobierno" que engloba un concepto más amplio referido al acceso que tienen los ciudadanos a la información, el cual finalmente lleva a lograr una rendición de cuentas y transparencia. Dentro de esta misma definición Mitchell (1998) destaca que esta palabra hace referencia a una demanda de información, la capacidad que tienen los ciudadanos de obtener esta información por parte del Gobierno y ONG lo que estaría indicando que no es suficiente con una simple liberación de la información por parte de las distintas empresas que se incorporan a este concepto de divulgación; sino que también debe existir una participación activa por parte de los interesados que pueden provenir de distintos sectores tanto internos como externos para que así se haga realmente aplicación de lo que se refiere este concepto (Sandoval, 2015). 
Teniendo en cuenta estas definiciones se puede demostrar que respecto de la información que abarcara este trabajo, información medioambiental en un marco de responsabilidad social es divulgada por parte de las distintas empresas en sus páginas web con el objetivo de transparentar a la sociedad su comportamiento en el contexto de cómo se están comportando ellas con su entorno, así sometiéndose a una especie de evaluación por parte de todo aquel que actúe como receptor de esta información en los ámbitos sociales, económicos y medio ambientales.

\section{Información financiera y no financiera}

Podemos definir información financiera como el conjunto de datos presentados de manera sistemática, que es medida y expresada en términos monetarios, que describen la situación financiera, los resultados de las operaciones y flujos de efectivo y otros aspectos relacionados con la obtención y el uso. A partir de esta información podemos obtener las razones o ratios financieras que permiten analizar variables de la empresa como su necesidad de flujo monetario, liquidez de la empresa, apalancamiento financiero, gastos fijos y facilitan la evaluación de eficiencia de la gestión de sus activos, pasivos y patrimonio durante el transcurso del periodo que está mostrando la organización, más allá de la simple toma de decisiones de los distintos usuarios tanto externos como internos (Gómez et al., 2017). Según Jones (2003) las organizaciones son administradores de los activos que controlan, ya sean estos los activos financieros o no financieros. Existe una premisa subyacente de que las organizaciones son responsables ante la sociedad en general, así como ante las partes interesadas por su gestión de impactos el entorno. Deben tener en cuenta el medio ambiente de forma altruista, pero también de forma al interés propio.

En el caso de la Información no financiera o cualitativa, según el Financial Accounting Standard Board (FASB, 2001) es aquella que no está reflejada en los estados financieros tradicionales, pudiendo comprender información obligatoria y voluntaria y su objetivo es capturar datos que informen sobre aquellos aspectos del negocio que no están reflejados en el juego de estados financieros básicos. De otro modo se podría definir como la información no monetaria. Este tipo de información suele estar principalmente en el ámbito voluntario; es decir la que no se está obligada a emitir por la normativa legal o contable. De acuerdo con Malgioglio et al. (2012) 
dentro de esta información no financiera se pueden distinguir dos clases; "Memorias o Informes de Sostenibilidad" y los "Informes de Gobiernos Corporativos". Sobre las Memorias o Informes de Sostenibilidad, la mayoría de las empresas en Latinoamérica emplean lineamientos del Global Reporting Initiative (GRI), y según las estadísticas que refleja el Corporate Register en su página web, en Chile al 2017 existían 213 organizaciones que emiten estos reportes y cada año existe un aumento de la cantidad de reportes emitidos en el país. En cambio, en los Informes de Gobiernos Corporativos, en Latinoamérica es utilizado principalmente el White Paper, que usa principios de Gobiernos corporativos de la OCDE 2016 como marco de trabajo conceptual y el cual se creó en la Mesa redonda para Gobierno Corporativo Latinoamericano, la cual está organizada por la Organización para la cooperación y desarrollo económico, Banco mundial, Corporación Financiera Internacional y socios regionales clave tanto del sector público como privado. Este documento no es obligatorio, aunque examina la importancia de un buen gobierno corporativo y fija recomendaciones, que con el tiempo se están enmarcando en regulaciones del regulador chileno (Comisión para el Mercado Financiero, reconocida como CMF Chile) como Normas de Carácter General.

\section{Divulgación de información a través de Internet}

En la actualidad Internet tiene un rol fundamental para publicación de información accesible para todos los usuarios, por esto pasa a ser fundamental que las empresas mantengan actualizadas sus páginas web que es el medio donde esta información tiende a ser divulgada, y como muestra el mismo reporte nombrado con anterioridad (Reporting matter del Consejo Empresarial Mundial para el Desarrollo Sostenible, también conocido por su sigla en inglés como WBCSD), las empresas están tendiendo hacia la digitalización de sus reportes mostrando que un 53\% de las empresas incluye la mayor parte de su contenido en línea para complementar sus informes en formato pdf.

El uso de Internet como herramienta de comunicación de información crece cada vez más debido a las múltiples ventajas y oportunidades que trae su uso, además de que reduce de forma importante los costos asociados, generando informes más "rentables" y trae un mayor grado de confianza en las firmas que divulgan información en sus páginas web a través de este medio, ya 
que llegan de una mejor forma a sus stakeholders y pueden proporcionar una gran cantidad de información de forma rápida y accesible (Romero, 2014).

Una de las incorporaciones que tuvo Chile a este medio fue en el 2012, la que en ese entonces se llamaba Superintendencia de Valores y Seguros (SVS) y actualmente CMF Chile emitió una norma de carácter general $\mathrm{N}^{\circ} 341$ (NCG 341) con el objetivo de crear un mecanismo de autorregulación de los estándares de difusión de información en los Gobiernos Corporativos adoptados por las Sociedades anónimas abiertas, suministrando al mercado más y mejor información con respecto a las prácticas del Gobierno Corporativo. Esta norma contiene prácticas y principios que son voluntarios; sin embargo, la respuesta al cuestionario es obligatorio y debe ser publicada en la página web de las sociedades abiertas (García, 2014). Con el tiempo dicha norma se separa en dos diferentes normas, la N³85 y 386 estableciendo criterios más específicos en materias de gobernanza y la otra sobre materias relacionadas con ESG (Environmental, Social y Governance).

\section{Objetivos de Desarrollo Sostenible (ODS)}

Cada día se evidencia una mayor relevancia sobre el comportamiento de las empresas con su entorno que puede tener repercusiones en cómo se desempeñan en su rendimiento, esto es lo que Moneva (2007) señala como la principal motivación para escuchar a sus stakeholders, es que estos invertirán según el comportamiento social responsable. A pesar de que esto puede ser una motivación como la reducción de costos al tener procesos más eficientes, las mejoras de su imagen corporativa y todo esto trae beneficios para su entorno al motivar a las firmas a acciones como por ejemplo reutilización de residuos, contratación laboral responsable, igualdad de género y ayudas sociales que muchas veces salen de las operaciones propias de la empresa.

Dentro de este contexto se decide el lanzamiento 17 Objetivos de Desarrollo sostenible (ODS) en septiembre del 2015 en una Cumbre del Desarrollo Sostenible donde asistieron 150 jefes de Estado y Gobierno aprobando la Agenda 2030 de aplicación Universal al 1 de enero del 2016. Estos objetivos han sido tomados en cuenta de forma muy aceptada por las empresas (Objetivos de Desarrollo Sostenible, 2019), debido a que estas de a poco los han ido incorporando dentro de sus informes de sostenibilidad, poniéndose desafíos a sí mismas para el logro de estos, según 
como la naturaleza de sus operaciones tiene mayor o menor influencia en determinados objetivos. Un buen resumen es la Figura 1 siguiente con los aspectos principales de los ODS:

\section{Figura 1}

Objetivos de desarrollo sostenible según la ONU
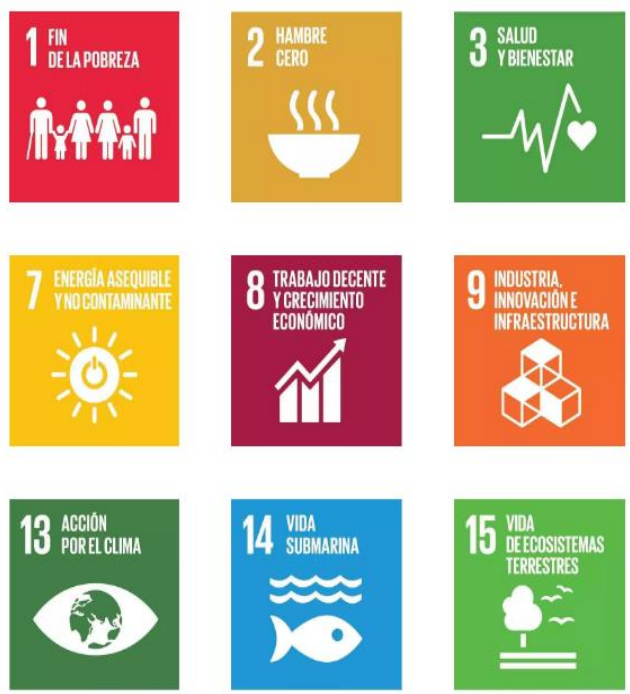
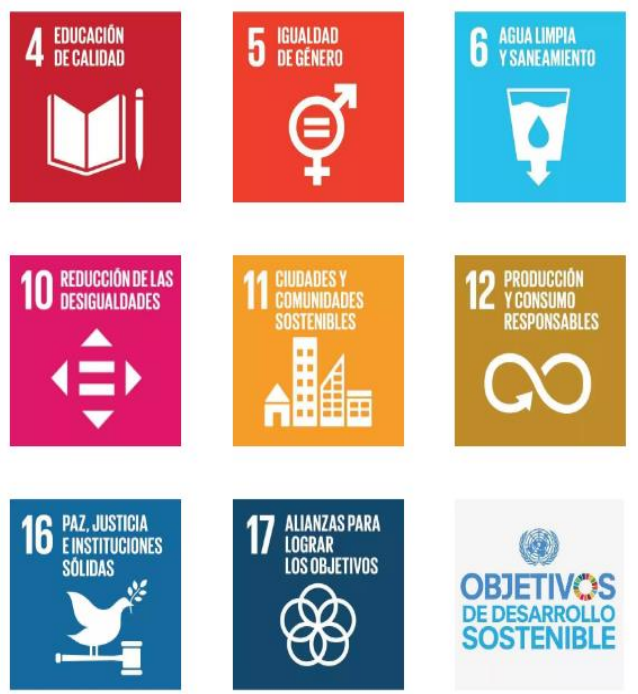

Fuente: Organización de Naciones Unidas (ONU, 2015).

\section{Revisión de Literatura}

En el último tiempo ha existido un aumento por la preocupación del entorno de las distintas empresas que desarrollan sus actividades, por lo que existe cada vez una mayor exigencia hacia ellas al momento de pedirle una mayor transparencia de su información, para "rendir cuentas a la sociedad" (accountability), capturando así una posición de "legitimidad", exigiéndoles más allá del cumplimento de lo que es meramente legal, también un valor agregado incorporando un trato moral que genere un bienestar social transparentando información relevante a la comunidad (Gray et al., 1997) siendo estas las principales motivaciones para hacer informes de sostenibilidad por parte de las empresas (Cunha y Moneva, 2018).

No es fácil relacionar la dinámica del accountability con una sola teoría, ya que pueden ser diversos los enfoques y por lo tanto, la adopción de una teoría para tratar de explicarlo puede ser limitado. Además, el fenómeno que parte de una necesidad propiamente del sector público, 
encontró sentido en la información pública del sector privado, especialmente de los mercados financieros. Es así como la idea de la rendición de cuentas, también recocida por "divulgación obligatoria y voluntaria" fue adoptando el término como una acepción más amplia y no sólo vinculada a un sector en particular. Por lo tanto, con los años, los conceptos de revelaciones, divulgaciones, disclosure y reporting por las expresiones habitualmente usadas en inglés, fueron todas teniendo cabida en la idea del accountability e incluso en la parte que se refiere a revelaciones, la expresión compliance también tiene espacio bajo la idea de la rendición de cuentas (en parte), aunque esta última abarca un mayor espectro de situaciones y ámbito de aplicación.

Diversos autores han estudiado qué factores son los que se relacionan con la divulgación de información de RSE, como Briano y Rodríguez (2013) que por medio de un índice de transparencia corporativa (ITC) de las empresas españolas del IBEX-35 llegan a la conclusión que existía una relación positiva entre el tamaño de la empresa y la independencia del consejo; sin embargo este país puede ser un caso distinto al contexto latinoamericano, debido que España a diferencia del contexto latino han existido marcos normativos o estándares desde la "Ley Aldama" (Santoloría y Bernal, 2008) hasta la actual Ley 11/2018 en materias no financieras obliga a publicar información por medio de su páginas web, diferente al contexto latino menos regulado.

Según Gómez y García (2009) que estudiaron a Chile, México y Colombia afirman que el nivel de divulgación de información social era bajo, destacando que lo más divulgado es la información a la comunidad y lo menos es acerca de los consumidores. Otros estudios hablan de que los índices más divulgados son respecto de los ingresos e inversiones totales y los menos sobre la corrupción en Brasil, lo que coincide con estadísticas de la ONU (Oliveira et al., 2009). Según diferentes trabajos, como en Larrán y Giner (2002) entre más selectiva sea la muestra, la extensión relativa de la información de la página web es más elevada. En la divulgación de información el coste de distribuir información por internet es constante e independiente del número de usuarios, por lo que empresas de gran tamaño están más interesadas de utilizar este medio y distribuir una mayor cantidad de información (Babio et al., 2001). 


\section{Materiales y Método}

Esta investigación se basa en un análisis empírico, descriptivo, el cual se centra en empresas chilenas emisoras de valores pertenecientes a sectores empresariales productivos con mayor impacto medioambiental, debido al grado de relación que poseen este tipo de empresas con el ámbito de la gestión ambiental por el uso directo de los recursos naturales en sus operaciones.

Las empresas seleccionadas se obtuvieron de la página oficial de la CMF Chile, el total de empresas cotizadas que arrojó el sitio fueron 367 de las cuales se descartaron 242 ya que no se encontraban ligadas directamente a empresas productivas teniendo un menor impacto en el medio ambiente, tampoco presentan en sus productos o servicios emisiones atmosféricas, intervención dañina en la flora y fauna, sin embargo, esto no quiere decir que no dejen huella en términos ambientales. Entre los sectores descartados están las instituciones financieras, educacionales, sociedades de inversiones, clubes deportivos, servicios de salud del sector privado y compañías telefónicas.

Luego del primer filtro quedaron 125 empresas, de las cuales 7 no tenían sitios web activos en el momento de la revisión, dando como resultado 118 empresas. Sin embargo, esta cantidad se redujo en la revisión de páginas web, por el hecho de que algunas empresas controladoras compartían sitios con sus filiales o en los casos que una empresa más grande engloba varias que formaban parte de ella.

Las 107 empresas cotizadas chilenas seleccionadas son: servicios básicos que se componen por agua, gas y electricidad representando un $28,97 \%$ de las empresas analizadas (31 empresas); manufactura que agrupa empresas del área alimentaria, cementos, manufactura y vinícola, representando un 24,3\% (26 empresas); constructoras que son el 6,54\% (7 empresas); agrosilvicultura 8,41\% (9 empresas); mineras, que agrupan tanto el área minera como del petróleo y metalúrgica, que son el 7,48\% (8 empresas); transporte y almacenamiento con un 12,15\% (13 empresas); pesca un 7,48\% ( 8 empresas); y finalmente otros con un 5,61\% (3 empresas). 
Se realizó la evaluación con base a una lista de chequeo compuesta por 25 variables las cuales analizan el contenido que presente en los sitios web de las entidades organizadas en 4 sub índices y un índice total ( 5 en total), los cuales se pueden ver en la Tabla 1.

\section{Tabla 1}

Características de los índices

\begin{tabular}{lcl}
\hline \multicolumn{1}{c}{ Índice } & $\begin{array}{c}\text { No. } \\
\text { variables }\end{array}$ & \multicolumn{1}{c}{ Características } \\
\hline General (IG) & 3 & $\begin{array}{l}\text { Empresa divulga información, ya sea mediante su } \\
\text { memoria anual, informe sustentabilidad o reporte } \\
\text { integrado. }\end{array}$ \\
\hline $\begin{array}{l}\text { Medioambiental } \\
\text { (IM) }\end{array}$ & 5 & $\begin{array}{l}\text { Centrado en cómo se comporta la empresa con su } \\
\text { entorno tanto externo e interno y que ha realizado para } \\
\text { disminuir sus externalidades negativas. }\end{array}$ \\
\hline Social (IS) & 11 & $\begin{array}{l}\text { El comportamiento de la empresa ante la comunidad y } \\
\text { sobre las condiciones de los trabajadores en la } \\
\text { empresa. }\end{array}$ \\
\hline $\begin{array}{l}\text { Gobierno corporativo } \\
\text { (IGC) }\end{array}$ & 6 & $\begin{array}{l}\text { Demuestra que tan transparente es el gobierno } \\
\text { corporativo de la empresa a través de su sitio web. }\end{array}$ \\
\hline Índice total (ITOT) & 25 & \begin{tabular}{l} 
Promedio simple de todos los índices parciales. \\
\hline
\end{tabular}
\end{tabular}

Fuente: elaboración propia.

Refiriéndonos a los ODS podemos indicar que principalmente se abarcó la No. 5 "Igualdad de género", No. 6 "Agua limpia y saneamiento", No. 7 "Energía asequible y no contaminante”, No. 8 "Trabajo decente y crecimiento económico", No. 9 "Industria, innovación e infraestructura", No. 10 "Reducción de desigualdades", No. 11 "Ciudades y comunidades sostenibles", No. 12 "Producción y consumo responsable" y No. 15 "Vida de ecosistemas terrestres". Dentro de estos objetivos los de mayor cumplimiento es el No. 9 y No. 11 al revelar información de inversión en procesos sostenibles que la compone la variable número 5, dentro del mismo aspecto medio ambiental, no se observó gran divulgación de las variables relacionadas con el objetivo No. 6 y No. 15 quienes se enfocan en la huella de carbono y emisiones atmosféricas.

La evaluación de las empresas se hizo por medio de puntajes de 0;0,5 y 1, siendo respectivamente "No divulga", "Divulga parcialmente" (referido cuando la información es 
presentada de forma desactualizada, anterior al 2018) y "Si Divulga", dando un puntaje determinado en donde el total es 24 puntos, en el caso de presentar todas las variables y convertida a una escala de 10 puntos máximo. Además, se revisó la información contable de las empresas buscando aspectos ambientales y sociales informados en notas explicativas, el cual se realizó a partir de la revisión de las memorias y estados financieros. Trabajos previos que ha servido como referencia en la aplicación de esta metodología, se pueden consultar en Morales y Jarne (2009), Morales (2012) y Mohamed y Basuony (2014).

\section{Resultados}

El análisis de los 107 sitios web arrojó un 9,67 como mejor resultado, correspondiendo a la compañía Aguas Andinas, en una escala de 1 a 10. Cumple con todas las variables de la lista de chequeo en un $100 \%$ excepto el de Gobierno Corporativo, donde falla en no poseer un modelo para la prevención de delitos. Por el contrario, la empresa con menor puntuación obtuvo un 0,50, Invertec Foods S.A., la cual a diferencia de Aguas Andinas sólo cumple con tener un canal de comunicación con sus clientes en el índice social, algo bastante básico en los sitios web, ya que un $90,65 \%$ cumplen con esta variable. Los resultados por índice para toda la muestra se presentan a continuación en Tabla 2:

\section{Tabla 2}

Resultados por índice para toda la muestra

\begin{tabular}{ccccccc}
\hline Índices & N & Media & Mediana & Mín & Máx & $\begin{array}{c}\text { Desviación } \\
\text { estándar }\end{array}$ \\
\hline IG & 107 & 4,80 & 3,33 & 0,00 & 10,00 & 2,35 \\
IMA & 107 & 3,37 & 2,14 & 0,00 & 10,00 & 3,24 \\
IS & 107 & 4,44 & 5,00 & 0,00 & 10,00 & 2,35 \\
IGC & 107 & 5,94 & 6,67 & 0,00 & 10,00 & 2,59 \\
ITOT & 107 & $\mathbf{4 , 1 9}$ & $\mathbf{3 , 8 3}$ & $\mathbf{0 , 4 0}$ & $\mathbf{9 , 6 0}$ & $\mathbf{2 , 1 1}$ \\
\hline
\end{tabular}

Fuente: elaboración propia.

Analizando cada índice comenzando por el General (IG), un 93,46\% (100 empresas) revela información relativa a sustentabilidad por medio de su memoria anual, de estas, 10 presentan además, reporte integrado e informe de sustentabilidad, y 28 informe de sustentabilidad junto a la memoria; respecto al informe de sustentabilidad es presentado por un 37,38\% y el Reporte 
integrado sólo por un 10,28\% de las empresas (10 empresas). De estas un 89,92\% presentan sus informes de auditoría por las 4 auditoras más grandes conformadas por Deloitte, PWC, EY y KPMG, un 36,54\% presenta informe de sustentabilidad estandarizado en normativa GRI y sólo un $5,88 \%$ bajo normativa IIRC. No es menor el asunto relativo a certificaciones o auditorías de la información suministrada en web, ya que el asunto de la fiabilidad es una discusión vigente que data desde el origen de las revelaciones voluntarias (AECA, 2002). De hecho si observamos en la muestra que empresas divulgan informes con algún tipo de certificación asciende a 5,06, mientras que las que no se sitúan en un promedio de 3,65, en la escala de 1 a 10.

Dentro del Índice Social (IS) un 50\% de las empresas cumple con un $25 \%$ de las variables o menos y el índice de Gobierno Corporativo (IGC) un 50\% de las empresas cumple un 66,67\% de las variables o más.

En la Tabla 2 se pueden observar los 4 índices parciales analizados a partir de su promedio obtenido, es el IGC es el de mejor prospectiva de revelaciones obedeciendo a una temprana obligatoriedad del regulador chileno sobre prácticas de transparencia en materias de gobernanza corporativa dirigidas al mercado, seguido por la disponibilidad general de reportes en web (IG), luego los aspectos sociales (IS) que contienen los aspectos sobre prácticas laborales y finalmente los relativos al medioambiente (IM). Todos poseen medianas muy distintas, siendo la mayor la del IGC que es el mejor valorado y la menor IMA, la que es muy cercana a su promedio, además de ser el de menor valoración; otras observaciones son que el IS tiene una mediana cercana a su promedio dando cuenta de una mayor homogeneidad en los datos; el IMA tiene una mediana mucho menor que su promedio y una mayor desviación estándar, lo que sería un indicador de mayor dispersión en la calidad de las revelaciones realizadas en las empresas chilenas en temas medioambientales.

Finalmente se puede ver que los índices con el grupo de empresas mejor evaluados son el social y el de gobierno corporativo, como se puede apreciar ya que presentan las cajas más altas. Además, y complementando todo lo anterior se resume en la Figura 2 de cajas y bigotes que permite una mayor comprensión de lo ya analizado bajo una mirada exploratoria de los índices. 


\section{Figura 2}

\section{Diagrama de caja por índices}

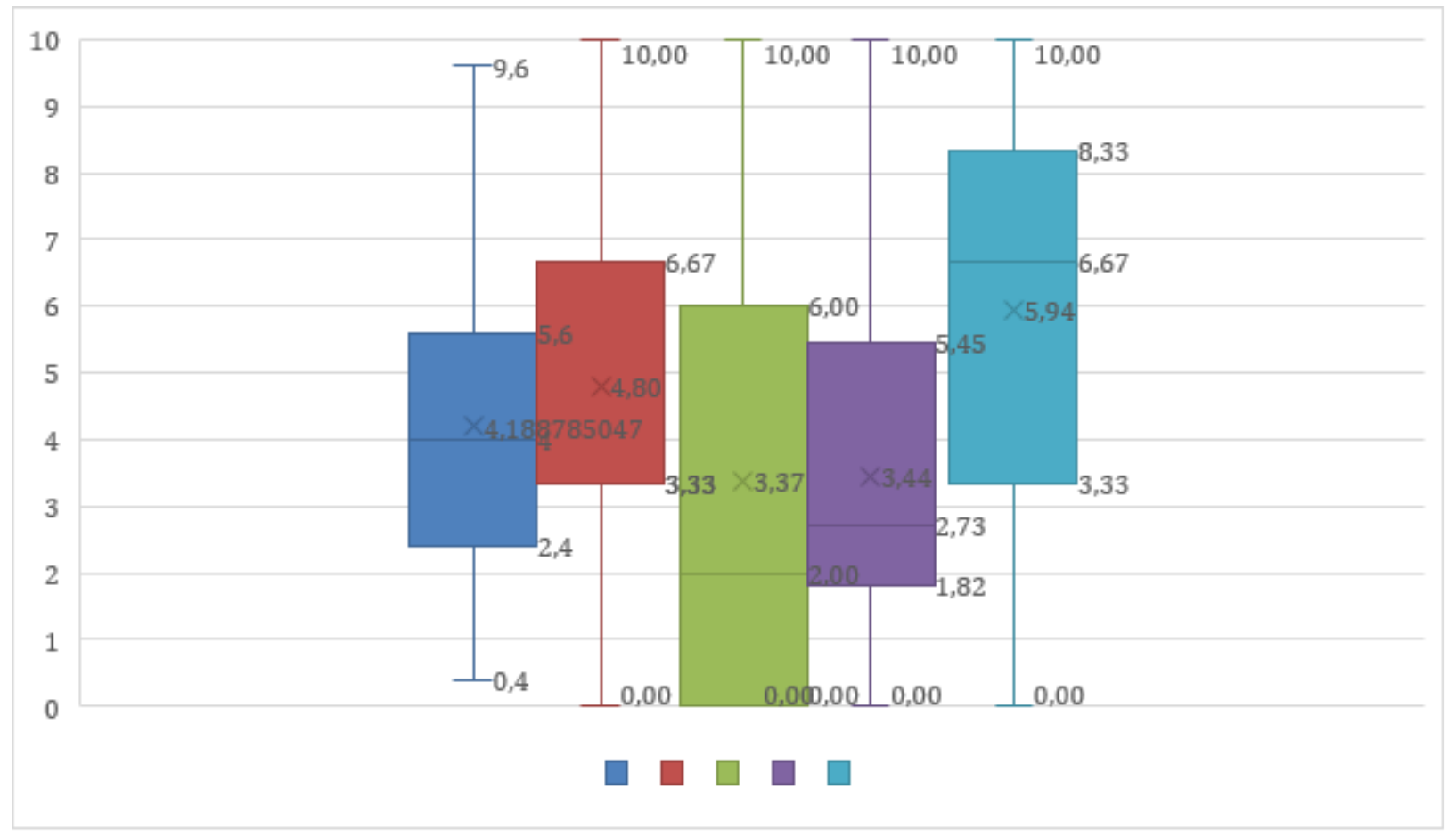

Fuente: elaboración propia.

\section{Análisis por sector empresarial}

En este apartado se analizan los distintos sectores empresariales para determinar si esta variable dependiente influye en los resultados obtenidos. Los sectores analizados se han clasificado según la naturaleza de las empresas, siendo descritas mediante un análisis gráfico mediante el uso de diagrama de cajas utilizando para su desarrollo 5 índices (4 índices parciales y el índice total). A continuación, se entregan datos estadísticos para visualizar la tendencia, valores encontrados en la mitad, medidas de dispersión respecto a la media, mínimo, máximo y rango, quedando demostrado en la Tabla 3.

Se puede observar que el sector con la mayor media y mediana es la minería, pero además es importante señalar que posee la segunda mayor desviación estándar; y a pesar de poseer los valores más altos en estas medidas, el sector que tiene la empresa con la nota máxima es el de servicios básicos, específicamente el subsector del agua con la empresa Aguas Andinas que es 
una empresa destacable, pero no podemos decir lo mismo del subsector agua debido a que presenta el segundo rango más amplio y una alta desviación estándar indicando que los puntajes obtenidos por las empresas tienen una alta variabilidad.

\section{Tabla 3}

Tabla análisis descriptivo por sector

\begin{tabular}{lcccccc}
\hline \multicolumn{1}{c}{ Sector } & Media & Mediana & $\begin{array}{c}\text { Desviación } \\
\text { Estándar }\end{array}$ & MIN & MAX & Rango \\
\hline Agro silvicultura & 3,03 & 3,17 & 1,37 & 1,50 & 5,86 & 4,36 \\
Agua & 4,52 & 4,45 & 2,16 & 1,83 & 9,67 & 7,83 \\
Electricidad & 4,40 & 4,74 & 2,08 & 1,50 & 8,38 & 6,88 \\
Gas & 4,23 & 5,93 & 1,72 & 2,67 & 6,69 & 4,02 \\
Manufactura & 4,66 & 5,05 & 2,39 & 0,50 & 8,55 & 8,05 \\
Constructoras & 4,19 & 4,81 & 1,38 & 2,45 & 5,48 & 3,02 \\
Minería & 5,49 & 6,21 & 2,27 & 2,50 & 7,76 & 5,26 \\
Pesca & 3,71 & 3,33 & 1,87 & 1,62 & 7,14 & 5,52 \\
Transporte y & 3,51 & 2,90 & 1,76 & 1,50 & 6,60 & 5,10 \\
Almacenamiento & 4,10 & 4,14 & 1,95 & 2,17 & 5,93 & 3,76 \\
Holdings & 2,48 & 2,48 & 0,91 & 1,83 & 3,13 & 1,29 \\
Otros & & & & & & \\
\hline
\end{tabular}

Fuente: elaboración propia.

Respecto a la media y mediana más baja la tienen la categoría otros; sin embargo hay que tener en cuenta que sólo está compuesto por los datos de dos empresas, por lo que no influye tanto en el resultado final, mientras que el sector más bajo en la medición de la divulgación es el de agro silvicultura el cual contiene datos de 9 empresa; además hay que tener en cuenta que dentro del análisis general posee la menor desviación estándar y uno de los más bajos rangos de datos; por lo tanto se podría decir que los valores del promedio son más representativos de los valores que realmente son tomados.

Un dato importante a destacar es que la cantidad de empresas por sector varía y esto puede influir en los resultados obtenidos, por ejemplo, en el sector Otros como ya se mencionó anteriormente se puede explicar la desviación más baja debido a que sólo se están contabilizando valores de 2 empresas y en contraste a esto el sector manufactura que está compuesto por datos de 26 páginas y tiene el valor más alto de desviación. 
Como una forma de verificar si las diferencias observadas en el análisis descriptivo de la divulgación basada en ODS y medidas a partir de los índices de medición en las web corporativas e informes suministrados en internet poseen diferencias significativas en dos agrupaciones que nos parece de interés, hemos propuestos las siguientes hipótesis nulas para oriental el trabajo:

SECTOR. Ho: No existen diferencias significativas entre los sectores económicos respecto de las divulgaciones de aspectos relativos a las ODS según la medición de este estudio.

CERTIFICACIONES. Ho: No existen diferencias significativas entre las empresas con información ambiental certificada y las que no poseen certificaciones respecto de las divulgaciones de aspectos relativos a las ODS según la medición de este estudio.

Para la comprobación estadística de las hipótesis aplicamos la prueba no paramétrica de KruskalWallis en el caso de la variable sector (al ser varios grupos de empresas) y la U de MannWhitney en el caso de la variable certificaciones (dos agrupaciones de empresas) de tal forma de verificar si los estadísticos confirman la existencia o no de diferencias significativas, cuyos resultados podemos observar en la siguiente Tabla 4:

\section{Tabla 4}

Estadísticos a partir de la prueba de Kruskal-Wallis

\begin{tabular}{|c|c|r|r|r|r|r|}
\hline \multirow{4}{*}{ Hipótesis } & Estadísticos & ITOT & IG & \multicolumn{1}{c|}{ IMA } & \multicolumn{1}{c|}{ IS } & \multicolumn{1}{c|}{ IGC } \\
\hline \multirow{3}{*}{ SECTOR } & H de Kruskal-Wallis & 11,223 & 4,894 & 13,345 & 13,587 & 13,503 \\
\cline { 2 - 7 } & $\mathrm{gl}$ & 9 & 9 & 9 & 9 & 9 \\
\cline { 2 - 7 } & Sig. asintótica &, 261 &, 843 &, 148 &, 138 &, 141 \\
\hline \multirow{2}{*}{ CERTIFICACIONES } & $\mathrm{U}$ de Mann-Whitney & 677,000 & 1050,000 & 717,500 & 560,500 & 1120,000 \\
\cline { 2 - 7 } & & $-4,451$ & $-2,297$ & $-4,335$ & $-5,226$ & $-1,660$ \\
\cline { 2 - 7 } & Sig. asintótica &, $000 * * *$ &, $022 * *$ &, $000 * * *$ &, $000 * * *$ &, $097 * *$ \\
\hline
\end{tabular}

$* * *<0,01 * *>0,01 \quad y<0,05 *<0,1$

Fuente: elaboración propia. 
Los resultados arrojaron que a nivel de sector (como variable independiente) no existen diferencias significativas en los niveles de divulgación sobre aspectos contenidos en los ODS de la forma como los hemos medido. Por lo tanto, se acepta la hipótesis nula de igualdad entre los sectores. Lo anterior es interesante, ya que puede observarse a las empresas chilenas desde una misma perspectiva regulatoria o de promoción de buenas prácticas en temas de accountability al ser un grupo relativamente homogéneo en su comportamiento de prácticas de revelaciones en materias de sostenibilidad. Lo anterior considerando que en la selección de la muestra, se priorizaron aquellas que tenían mayores impactos medioambientales de acuerdo con su actividad económica.

Por el contrario, el análisis por la variable independiente fue diferente al sector, ya que aquí si observaron diferencias significativas sobre la divulgación realizada en los grupos de empresas separados según las que ofrecen información de certificar o auditar sus informes o informaciones de sostenibilidad versus las que no lo hacen. Por lo tanto, se rechaza la hipótesis nula de igualdad entre los grupos de empresas. El nivel de significatividad para rechazar $H_{0}$ es en 4 índices del nivel del 1\% (muy significativo) mientras que sólo en el índice sobre gobernanza corporativa (IGC) es menos fuerte el estadístico pero igual válido para ser rechaza la hipótesis, lo que tiene sentido en el contexto que la normativa que promueve la mayor transparencia de informas elementos de gobernanza en Chile, ya tiene varios años, provocando un efecto de mayor similitud entre las empresas. En este sentido, Oliveira et al. (2009) son de la idea que los responsables de la formulación de políticas tal vez deseen considerar medidas para promover reportes voluntarios de información de responsabilidad corporativa. Se pueden avanzar para estandarizar tal información alrededor de un solo conjunto de indicadores de alta calidad, sugiriendo como un conjunto más amplio (cuantitativos e indicadores descriptivos) encontrados en las directrices de la Global Reporting Initiative.

Al observar los rangos promedios en el caso de las empresas que mantienen algún tipo de certificación en las informaciones divulgadas, comprobamos que en todos los índices parciales y en el índice total, son mayores en las empresas que poseen certificaciones por sobre las empresas que no, por lo tanto la relevancia que tiene para las empresas de la muestra el hecho de estar certificadas, tiene una relación directa y significativa con los niveles de divulgación en web 
sobre aspectos de sostenibilidad. Lo anterior va de acuerdo con lo que promueve y observa AES (2021) parafraseando a Deloitte, en el sentido que las agencias evaluadoras externas son prestadores de servicios que desempeñan un papel principal a la hora de garantizar la seguridad de la información sobre sostenibilidad y pueden evaluar, verificar y/o certificar que un producto financiero sostenible específico está alineado con los estándares o pautas de sostenibilidad requeridos y la regulación existente, o hacer recomendaciones frente a las mejores prácticas.

\section{Análisis de información medioambiental e información contable}

En este último apartado se referirá al análisis de la existencia de información medio ambiental de las empresas por medio de la revisión de las notas explicativas en sus EEFF para determinar si existe divulgación por parte de estas de información medio ambiental.

Comenzamos este análisis por medio de los resultados generales obtenidos de la revisión de las memorias anuales y/o EEFF, dependiendo de donde fueron publicadas las notas explicativas en los respectivos sitios web de las empresas; de estos se puede observar que en general existe una mayor divulgación con un $83,18 \%$ que equivale a 89 empresas, versus a un 16,82\% (16 empresas) que no cumplen con tener una nota explicativa en relación al medioambiente.

\section{Tabla 5}

Clasificación de gastos

\begin{tabular}{lcc}
\hline \multicolumn{1}{c}{ Clasificación Gasto } & No. de empresas & \% de empresas \\
\hline No presenta gastos & 17 & $19 \%$ \\
No detalla gastos & 6 & $7 \%$ \\
Gastos acústicos & 4 & $4 \%$ \\
Gasto gestión ambiental & 40 & $45 \%$ \\
Gastos energéticos & 3 & $3 \%$ \\
Gastos en RILES & 28 & $31 \%$ \\
Gasto de inversión en mejoras e infraestructura & 22 & $25 \%$ \\
Gastos reducción olores & 1 & $1 \%$ \\
Gastos emisiones & 9 & $10 \%$ \\
Gastos otros residuos (RISES) & 16 & $18 \%$ \\
\hline
\end{tabular}

Fuente: elaboración propia. 
De la Tabla 5 cabe señalar que, respecto a los datos arrojados en la recolección, se demuestra el interés de las empresas en realizar gestiones ambientales y en la peor calificación quien presenta gastos en reducción de olores, sólo una empresa. Además, son 6 las empresas que informan sobre gastos incurridos nombrando las operaciones realizadas, pero no indica el monto desembolsado, sin embargo, en el total de las empresas 17 presentan una nota explicativa señalando que no se incurrieron en gastos ya sea por su actividad o el gasto no haya sido relevante para llevarlo a resultado.

\section{Conclusiones}

Los objetivos de este trabajo de investigación fue determinar el grado de divulgación respecto a la información ambiental en las páginas web de 107 empresas cotizadas en la CMF, igualmente se observó dentro de sus estados financieros en la sección de notas explicativas sobre si presentaba aspectos relacionado a medio ambiente y finalmente se identificaron aspectos relacionados con los ODS. Además, la muestra de empresas analizadas presenta información en sus sitios web, pero no en la mayoría y no abarcando todas las variables analizadas; sobre todo al referirse al medioambiente y laboralmente es bastante escaso lo que se revelan en sus sitios corporativos.

En relación a temas medio ambientales tratados en la información contable de las empresas, se visualiza que en los EEFF las notas explicativas de medio ambiente concluyendo que la mayoría presenta esta nota, sin embargo, en algunas la presentan para indicar que dado su actividad no se incurrieron en gastos significativos o no consideraron relevante esta nota, no dándole la importancia que amerita el impacto que generan en su entorno las actividades normales de las empresas, hablando en estas de una manera muy general y no con el detalle necesario mostrando una realidad en donde pareciera que casi no generan externalidades negativas, lo que no se condice con cada industria seleccionada en esta investigación, justamente por la relación de sus operaciones con el entorno.

Este trabajo ha permitido llegar a la conclusión de que las empresas no tienen una gran divulgación en sus sitios web de las variables analizadas, sobre todo a lo que se refiere a temas 
laborales y medioambientales aunque sí con mayor frecuencia a los índices de temas sociales y de gobierno corporativo, pero igualmente en variables más bien genéricas como la identificación de sus miembros y canales de comunicación, en vez de temas más relevantes como cuál es su verdadero impacto en la comunidad o si existen políticas anticorrupción y soborno, las cuales demostrarían una verdadera transparencia de parte de las empresas; pudiéndose pensar que la información que eligen revelar muchas veces es pensada en que los pueda beneficiar o de otra manera que no los perjudique demasiado. La importancia de mejorar las prácticas de divulgación en temas de sostenibilidad, ODS y otros aspectos desde la empresa cotizadas es muy relevante, ya que van de la mano de la inversión responsable, como lo concluye también Panario et al (2021) desde la perspectiva del desarrollo de índices en Latinoamérica afirmando que es incipiente, y es importante fomentar y socializar dichas herramientas en los mercados de capitales regionales ya que los mismos pueden ser utilizados desde distintas perspectivas.

Una de las limitaciones en el desarrollo de este trabajo fue la recolección de información de forma visual, la cual es susceptible al error humano, siendo aquello un punto de gran importancia para justificar las posibles diferencias que pudieran hallarse respecto a la recolección de datos. También es importante indicar que la información varía en sus fechas desde el comienzo de esta investigación, por lo tanto, influye en el posible hallazgo de contenido distinto al expuesto. Por último, puede ser interesantes de considerar en trabajos futuros, analizar de manera más extendida la información que se encuentra en los informes de sustentabilidad y memorias y compararlas con lo que divulgan en sus sitios web; abarcar todas las empresas que cotizan en la bolsa e incluso comparaciones internacionales a partir del índice propuesto y finalmente realizar un análisis en mayor profundidad sobre las notas medio ambientales contenidas en los estados financieros bajo metodología de análisis cualitativo.

\section{Referencias}

(1) AECA [Asociación Española de Contabilidad y Administración De Empresas] (2002). Código de buenas prácticas para la divulgación de información financiera en Internet. (Ed) AECA. 
(2) AES (2021). "Creando valor sostenible: Hacia una divulgación ASG de calidad”. Informe con recomendaciones. https://aechile.cl/2021/05/27/aechile-presenta-el-estudio-creandovalor-sostenible-hacia-una-divulgacion-asg-de-calidad/

(3) Babio, A., Rosario, M., Vázquez, M., Flora, M. y Lopo, V. (2001). Voluntary Disclosure in Spain: an investigation of prepares perceptions. Paper presented at the 24th Annual Congress of the EAA. Athens, Greece.

(4) Briano, G. \& Rodríguez, L. (2013). Transparencia de la información corporativa en internet de las empresas del IBEX 35. Revista de contabilidad y dirección, 16, 187-208.

(5) Cabrera, J. (2008). La transparencia es clave para el futuro de nuestras organizaciones. Cabreramc. https://blog.cabreramc.com/2008/10/09/la-transparencia-es-clave-para-el-futurode-nuestras-organizaciones/

(6) Carqués, F. (2007). Una aproximación desde la teoría de la legitimidad a la información medioambiental revelada por las empresas españolas cotizadas. Spanish Journal of Finance and Accounting/Revista Española de Financiación y Contabilidad, 36(133), 97-121.

(7) Cunha, D. R. \& Moneva, J. M. (2018). La elaboración del informe de sostenibilidad: un estudio de caso. Rev. Bras. Gest. Neg. São Paulo v, 20(4), 533-549.

(8) EY (2017). "Can innovative corporate reporting build trust in a volatile world?: Finance 4.0 and the transformation of reporting and governance in a high-risk world". https://www.ey.com/Publication/vwLUAssets/ey-financial-accounting-advisory-servicesfaas-fourth-global-corporate-reporting-survey/\$FILE/ey-financial-accounting-advisoryservices-faas-fourth-global-corporate-reporting-survey.pdf

(9) FASB [Financial Accounting Standard Board] (2001). Improving business reporting: Insights into enchancing voluntary disclosures. Steering Committee Report. http://www.fasb.org/brrp/brrp2.shtml

(10)Foro de expertos RSE. (2005). Informe del foro de expertos en Responsabilidad Social de las Empresas. http://www.mitramiss.gob.es/es/sec_trabajo/autonomos/economiasoc/RespoSocEmpresas/foro_expertos/contenidos/INFORME_FOROEXPERTOS_RSE.pdf

(11) García, R. (2014). Norma de información sobre prácticas de gobierno corporativo en Chile. Superintendencia de Valores y 1-30. https://www.svs.cl/portal/estadisticas/617/articles-16379_doc_pdf.pdf 
(12) Gómez, A. \& García, M. (2009). La divulgación de información social en América Latina: el caso de México, Chile y Colombia. XV Congreso AECA.

(13) Gómez, C., Aristizabal, C. \& Fuentes, D. (2017). Importancia de la Información Financiera para el ejercicio de la Gerencia. Desarrollo Gerencial, 9(2), 88-101.

(14) Gray, R., Dey, C., Evans, R., Owen, D. \& Zadek, S. (1997). Struggling with the praxis of social accounting: Stakeholders, accountability, audits and procedures. Accounting, Auditing \& Accountability Journal, 10(3), 325-364.

(15) Hernández, J. y Yagui, V. (2021). Análisis de información y factores de desempeño ambiental y de economía circular en empresas peruanas. Revista De Investigación En Comunicación Y Desarrollo, 12(1), 37-52.

(16) IASC [International Accounting Standard Committee] (1999). Business reporting on the Internet. Discussion paper realizado por Lymer, A., Debrency, R., Gray, G. y Rahman, A. 103p.

(17) ICAS [Institute Chartered Accountants Scotland] (2018). Restoring trust and relevance for the future of corporate reporting. Corporate reporting vision - Straw man. Discussion paper. https://www.icas.com/_data/assets/pdf_file/0010/368461/VISION-STRAW-MAN4-June-2018-FINAL-PDF.pdf

(18) IFAC [International Federation Of Accountants] (2018). Information and communications technology literature review. IAESB. https://www.ifac.org/publicationsresources/information-and-communications-technology-literature-review

(19) KPMG (2020). The time has come. The KPMG Survey of Sustainability Reporting 2020. https://assets.kpmg/content/dam/kpmg/xx/pdf/2020/11/the-time-has-come.pdf

(20)Larrán, M. y Giner, B. (2002). The Use of the Internet for Corporate Reporting by Spanish Companies. The International Journal of Digital Accounting Research. 2(1) 55-85.

(21)Ley $\mathrm{N}^{\circ}$ 20.285, 2008 (2008/08/10). Ley Sobre Acceso a la Información Pública. https://www.leychile.cl/Navegar?idNorma=276363

(22) Malgioglio, J. M., Berri, A. M., Di Nenno, M. E., Campodónico, S., Fernández, R. N., Invernizzi, M. A. \& Tribolo, J. (2012). La importancia de la información no financiera para la toma de decisiones. Decimoséptimas Jornadas "Investigaciones en la Facultad" de Ciencias Económicas y

Estadística. 
https://rephip.unr.edu.ar/bitstream/handle/2133/7468/Malgioglio\%2C\%20Berri_\%20la\%20i mportancia\%20de\%20la\%20informacion.pdf?sequence $=3$

(23) Mitchell, R. B. (1998). Sources of transparency: information systems in international regimes. International Studies Quarterly, 42(1), 109-130.

(24) Mohamed, E. y Basuony, M. (2014). Determinants and characteristics of voluntary Internet disclosures in GCC countries. The International Journal of Digital Accounting Research 14 57-91.

(25) Moneva, J. M. (2007). ¿Es la responsabilidad social corporativa rentable para la empresa? $\begin{array}{llllll}\text { Revista de contabilidad } & y & \text { dirección. } & 7, & 55-76\end{array}$ https://www.academia.edu/2522061/_Es_la_responsabilidad_social_corporativa_rentable_p ara_la_empresa

(26) Morales, F. (2012). Análisis de la diversidad en la información empresarial divulgada por internet. Evidencia empírica en empresas europeas y latinoamericanas. INNOVAR. Revista de Ciencias Administrativas y Sociales. 22(44) 79-89.

(27) Morales, F. y Jarne, J. (2009). E-gobierno corporativo: evidencia en Chile sobre la divulgación de información en internet. Capic Review 7 (2009): 45-62.

(28) Naciones Unidas. (2019). Objetivos de Desarrollo Sostenible. https://www.un.org/sustainabledevelopment/es/objetivos-de-desarrollo-sostenible/

(29) OCDE [Organization For Economics Co-Operation And Development] (2015). G20/OECD Principles of Corporate Governance. OECD Publishing, Paris. http://www.oecdilibrary.org/docserver/download/2615021e.pdf?expires=1510531937\&id=id\&accname=gues t\&checksum=562E99A5A71983409EE56B05144F3DE6

(30) Oliveira, M. C., De Luca, M. M., Ponte, V. R. \& Pontes, J. É. (2009). Disclosure of social information by Brazilian companies according to United Nations indicators of corporate social responsibility. Revista Contabilidade \& Finanças, 20(51), 116-132.

(31) Organización de las Naciones Unidas [ONU]. (2018). ¿Cuánto le cuesta una hamburguesa al medio ambiente? https://news.un.org/es/story/2018/11/1445211

(32) Panario, M. P., Vior, I., Vior, Á., Rossi, A. \& Tallelis, C. (2021). Los índices bursátiles de sostenibilidad en América Latina y el Caribe: Relevamiento empírico. Revista Activos, 19(1). 
(33) Romero, F. T. (2014). Información del conocimiento organizacional a través de los informes anuales publicados en las páginas web de las empresas. Revista Española de Documentación Cientifica, 37(1), 031.

(34) Sandoval, R. (2015). Gobierno abierto y transparencia: construyendo un marco conceptual. Convergencia, 22(68), 203-227.

(35) Santolaria, C. J. S. \& Bernal, J. A. (2008). Un análisis de la transparencia informativa por medio de las páginas web corporativas. Contaduría Universidad de Antioquia. (52), 85-104.

(36) Vargas, J. (2008). Teoría institucional y neoinstitucional en la administración internacional de las organizaciones. Revista Científica Visión de Futuro. 10(2), 3-22.

Cómo citar este artículo: Morales, F., Vergara, D. y Jara, V. (2021). Divulgación de la información ambiental en páginas web en empresas cotizadas chilenas. Tendencias, 23(1), 200224. https://doi.org/10.22267/rtend.222301.188 